\title{
Percepção dos estudantes da Faculdade de Odontologia do Recife sobre o aplicativo ENDO UPE
}

\author{
Perception of Recife Dental School students about the ENDO UPE application \\ Percepción de los alumnos de la Facultad de Odontología de Recife sobre la aplicación de la ENDO
}

UPE

Recebido: 23/11/2021 | Revisado: 01/12/2021 | Aceito: 15/12/2021 | Publicado: 22/12/2021

\author{
Manoelle Augusta Bacelar \\ ORCID: https://orcid.org/0000-0003-2407-3143 \\ Faculdade de Odontologia do Recife, Brasil \\ E-mail: manoelle.bacelar@hotmail.com \\ Paulo Maurício Reis de Melo Júnior \\ ORCID: https://orcid.org/0000-0001-9926-5348 \\ Universidade de Pernambuco, Brasil \\ E-mail: Paulo.reis@upe.br \\ Sandra Maria Alves Sayão Maia \\ ORCID: https://orcid.org/0000-0003-3172-2332 \\ Universidade de Pernambuco, Brasil \\ E-mail: sandrinhasayao@hotmail.com \\ Bianca Maria Beserra Costa \\ ORCID: https://orcid.org/0000-0002-8132-9881 \\ Universidade de Pernambuco, Brasil \\ E-mail: bianca.bcosta@upe.br
}

\begin{abstract}
Resumo
Neste trabalho, objetivou-se avaliar a percepção dos estudantes da Faculdade de Odontologia do Recife (FOR) sobre o aplicativo ENDO UPE, como ferramenta de apoio ao ensino-aprendizagem. A amostra foi composta por 83 alunos do primeiro ao décimo período. O formulário online foi constituído por 16 itens de múltipla escolha e os resultados foram tabulados pela própria plataforma em planilhas (via Google Sheets), sendo visualizados em gráficos. Para tanto, os estudantes tiveram acesso ao convite da pesquisa através de um link do formulário online, que foi encaminhado pelo pesquisador por meio de mensagens aos grupos de WhatsApp das turmas de estudantes. Foi feita análise de frequência das variáveis (média e desvio padrão, valores máximo e mínimo). Foram utilizados os Softwares SPSS 13.0 (IBM, Armonk, NY, EUA) e Excel 2010 (Microsoft, Redmond, WA, EUA). Para verificar a existência de associações, utilizou-se os testes Qui-Quadrado e Exato de Fisher, para as variáveis categóricas considerando como nível de significância p-valor $\leq 0,05$ para um intervalo de confiança de $95 \%$. Os dados colhidos demonstram que os estudantes do curso de Odontologia da Faculdade de Odontologia do Recife (FOR) utilizaram de forma ampla o aplicativo ENDO UPE, o reconhecendo como ferramenta educacional do conteúdo de abertura coronária da Endodontia.
\end{abstract}

Palavras-chave: Endodontia; Aplicativos móveis; Materiais de ensino; Abertura coronária; Odontologia.

\begin{abstract}
This study aimed to evaluate the perception of students from the Recife School of Dentistry (FOR) about the ENDO UPE application as a tool to support teaching-learning. The sample was composed of 83 students from the first to the tenth period. The online form consisted of 16 multiple-choice items and the results were tabulated by the platform itself in spreadsheets (via Google Sheets) and visualized in graphs. For this, the students had access to the survey invitation through a link on the online form, which was forwarded by the researcher through messages to the WhatsApp groups of the students' classes. Frequency analysis of the variables was performed (mean and standard deviation, maximum and minimum values). SPSS 13.0 (IBM, Armonk, NY, USA) and Excel 2010 (Microsoft, Redmond, WA, USA) software were used. To verify the existence of associations, the chi-square and Fisher's exact tests were used for categorical variables, considering $\mathrm{p}$-value $\leq 0.05$ for a $95 \%$ confidence interval as the significance level. The data collected show that the students of the Dentistry course of the Faculty of Dentistry of Recife (FOR) widely used the ENDO UPE application, recognizing it as an educational tool for the content of coronal opening of Endodontics.
\end{abstract}

Keywords: Endodontics; Mobile apps; Teaching materials; Coronal opening; Dentistry. 


\begin{abstract}
Resumen
Este estudio tuvo como objetivo evaluar la percepción de los estudiantes de la Facultad de Odontología de Recife (FOR) sobre la aplicación ENDO UPE como herramienta de apoyo a la enseñanza-aprendizaje. La muestra estaba compuesta por 83 estudiantes del primer al décimo periodo. El formulario online constaba de 16 ítems de opción múltiple y los resultados fueron tabulados por la propia plataforma en hojas de cálculo (a través de Google Sheets), y visualizados en gráficos. Para ello, los alumnos tuvieron acceso a la invitación de la investigación a través de un enlace del formulario online, que fue remitido por el investigador a través de mensajes a los grupos de WhatsApp de las clases de los alumnos. Se realizó un análisis de frecuencia de las variables (media y desviación estándar, valores máximos y mínimos). Se utilizó el software SPSS 13.0 (IBM, Armonk, NY, EE.UU.) y Excel 2010 (Microsoft, Redmond, WA, EE.UU.). Para verificar la existencia de asociaciones, se utilizaron las pruebas de Chi-cuadrado y exacta de Fisher para las variables categóricas considerando como nivel de significación un valor $\mathrm{p} \leq 0,05$ para un intervalo de confianza del 95\%. Los datos recogidos muestran que los alumnos del curso de Odontología de la Facultad de Odontología de Recife (FOR) hicieron un amplio uso de la aplicación ENDO UPE, reconociéndola como una herramienta educativa para el contenido de apertura coronal de la Endodoncia.
\end{abstract}

Palabras clave: Endodoncia; Aplicaciones móviles; Material didáctico; Apertura coronal; Odontología.

\title{
1. Introdução
}

O uso das tecnologias de informação e comunicação (TICs) em sala de aula, para a área de Odontologia, auxiliam no aprendizado estudantil; nessa lógica, o estudante recebe a possibilidade de acessar informações rápidas e, na maioria das vezes, confiáveis sobre determinado conteúdo (Lopes et al., 2016). Esse processo, por sua vez, oferece um suporte que permite ao aluno atualizar os conhecimentos na matéria e acompanhar as novidades sobre várias temáticas, em tempo real, o que se mostra urgente para a sua preparação profissional no mercado de trabalho, cada vez mais tecnológico.

Diante da situação atual do País, no enfrentamento à pandemia da COVID-19 e de acordo com as recomendações da Organização Mundial da Saúde (OMS) sobre adoção de medidas de distanciamento e isolamento social, como forma de diminuir a propagação do vírus, torna-se pertinente que as ferramentas e estratégias de ensino-aprendizagem da Faculdade de Odontologia do Recife sejam aprimoradas para oferecer mais possibilidades de cenários e recursos ao ensino da Odontologia, de tal forma que a instituição se torne capacitada e incluída nessa nova realidade. (Costa et al., 2021)

$\mathrm{Na}$ área da saúde, as TICs além de possibilitarem a divulgação, disseminação e atualização do conhecimento, podem também apoiar tomadas de decisões clínicas desses profissionais, contribuindo inclusive com a elaboração de diagnósticos fidedignos e orientações/condutas terapêuticas qualificadas, destinadas aos pacientes/usuários (Lobo, 2015). Ressalta-se, ainda, que o acesso em tempo real e/ou remoto às informações colhidas nos TICs contribui para a solução de problemas/necessidades de saúde em diferentes regiões geográficas e à distância, promovendo ampla cobertura da assistência à saúde especializada, na maioria das vezes centralizada nos grandes centros urbanos (Barra et al., 2017).

No cenário tecnológico, destacam-se especialmente a utilização de aplicativos móveis (APPs - do inglês applications). Os APPs são conhecidos como um conjunto de ferramentas programadas para realizar e executar tarefas e atividades específicas. (Barra et al., 2017). A utilização de novas estratégias e metodologias ativas é definida como prioridade no país, e devem estar descritas nos projetos pedagógicos dos cursos como estratégias de ensino-aprendizagem, havendo necessidade da realização de projetos de intervenção para o desenvolvimento de novas experiências educacionais (Melo et al., 2018).

Diante desse contexto, um aplicativo educacional denominado "ENDO UPE - abertura coronária App" foi desenvolvido pelos docentes do curso de Odontologia da Faculdade de Odontologia de Pernambuco (FOP), vinculada à Universidade de Pernambuco, como ferramenta auxiliar no processo de ensino-aprendizagem dos procedimentos de abertura coronária dos elementos dentários. Tal aplicativo foi disponibilizado online por meio de um software/aplicativo para múltiplas plataformas digitais, como desktop e dispositivos móveis (smartphones ou tablets), com textos, imagens e vídeos demonstrativos. Em um primeiro momento, compreende-se que tal aplicativo buscou seguir a tendência nacional quanto à implementação da tecnologia como ferramenta impulsionadora do estudo acadêmico da Odontologia (Souza, 2018). 
O objetivo deste estudo foi avaliar a percepção dos estudantes de odontologia da Faculdade de Odontologia do Recife (FOR) sobre o aplicativo ENDO UPE, como ferramenta de apoio ao ensino-aprendizagem da endodontia.

\section{Metodologia}

Trata -se de uma pesquisa aplicada, descritiva, transversal, de levantamento e natureza quantitativa e qualitativa. O projeto de pesquisa foi encaminhado para o Comitê de Ética e Pesquisa, via Plataforma Brasil, mediante aprovação do sistema CEP/CONEP com parecer número 3.425.571. Foi elaborado pelos autores um questionário com 16 itens de múltipla escolha construído no formato do Google Forms, sobre o aplicativo ENDO UPE, disponibilizado através de link, via aplicativo WhatsApp para acesso ao questionário pelos estudantes. Os resultados foram tabulados pela própria plataforma em planilhas (via Google Sheets), podendo ser visualizados em gráficos. Foi feita análise de frequência das variáveis (média e desvio padrão, valores máximo e mínimo. Foram utilizados os Softwares SPSS 13.0 (IBM, Armonk, NY, EUA) e Excel 2010 (Microsoft, Redmond, WA, EUA). Para verificar a existência de associações, utilizou-se os testes Qui-Quadrado e Exato de Fisher, para as variáveis categóricas considerando como nível de significância $p$-valor $\leq 0,05$ para um intervalo de confiança de $95 \%$. Os discentes cursando o terceiro, quarto e quinto ano da graduação, que receberam orientação dos professores para o acesso ao aplicativo ENDO UPE na disciplina de Endodontia do curso de Odontologia Faculdade de Odontologia do Recife foram informados de todo o processo do estudo e, aqueles que aceitaram participar, assinaram o termo de consentimento livre e esclarecido (TCLE), antes de responderem o formulário. A amostra foi composta por 83 estudantes.

\section{Resultados}

Dos 83 estudantes, 33 estudantes cursavam o $3^{\circ}$ ano; 28 estudantes cursavam o $4^{\circ}$ ano e 22 estudantes cursavam o $5^{\circ}$ ano do curso de Odontologia da Faculdade de Odontologia do Recife (FOR). Todos os estudantes entrevistados utilizavam smartphones, 62 (74,7\%) utilizavam o aparelho da marca Apple, 12 (14,5\%) Samsung, 7 (8,4\%) Motorola e $2(2,4 \%)$ utilizavam outra marca. Em relação à quantidade de aplicativos utilizados no celular, 66 estudantes $(79,5 \%)$ disseram que têm mais de 7 aplicativos, 11 (13,3\%) entre 5 e 7, e $6(7,2 \%)$ disseram ter 1 a 4 aplicativos. As respostas de 77 estudantes (80,7\%) foram que utilizam aplicativos na área de saúde e de 71 (85,5\%) que utilizam aplicativos educacionais. A maioria dos estudantes participantes $(89,2 \%)$ conhecia o aplicativo ENDO UPE e $63(75,9 \%)$ responderam que o utilizavam, além de concordarem que esta ferramenta continuará sendo útil durante a vida profissional $(\mathrm{p}=0,041)$.

Logo, os dados demonstraram que grande parte dos alunos não só tinha conhecimento da ferramenta, como identificaram um uso benéfico do aplicativo, demonstrando suas potencialidades para além do espaço acadêmico.

Os dados relativos à frequência e local de utilização e demais situações de interação com o aplicativo ENDO UPE se encontram sumarizados na Tabela 1, abaixo disposta. 
Research, Society and Development, v. 10, n. 17, e138101723821, 2021

(CC BY 4.0) | ISSN 2525-3409 | DOI: http://dx.doi.org/10.33448/rsd-v10i17.23821

Tabela 1 - Dados da interação com o aplicativo ENDO UPE.

\begin{tabular}{ccc}
\hline Variáveis & n & $\%$ \\
\hline Frequência de utilização do aplicativo ENDO UPE & 1,6 \\
Sempre & 1 & 39,7 \\
Com frequência & 25 & 58,7 \\
Raramente & 37 & 22,2 \\
Local de utilização do aplicativo ENDO UPE & 39,8 \\
Clinica & 14 & 6,3 \\
Laboratório (aula prática) & 25 & 31,7 \\
Sala de aula (aula teórica) & 4 & \\
Todos & 20 & 96,8 \\
Compreensão do assunto ao utilizar o aplicativo & 3,2 \\
\hline Concorda & 61 & 2 \\
Discorda
\end{tabular}

Além do acesso ao aplicativo, a presença do professor é necessária nas aulas de abertura coronária

\begin{tabular}{ccc} 
Concorda & 63 & 100,0 \\
Discorda & 0 & 0,0 \\
\hline & O aplicativo é fácil de manusear & \\
Concorda & 61 & 96,8 \\
Discorda & 2 & 3,2 \\
\hline
\end{tabular}

Fonte: Autores.

A tabela acima demonstra, no formato de porcentagem, a resposta dos alunos entrevistados quanto à utilização do aplicativo. Em caso de dúvida sobre o conteúdo abertura coronária, prevaleceu o professor como a fonte de informação mais utilizada.

Figura 1 - Meios de informação consultados pelos estudantes sobre abertura coronária.

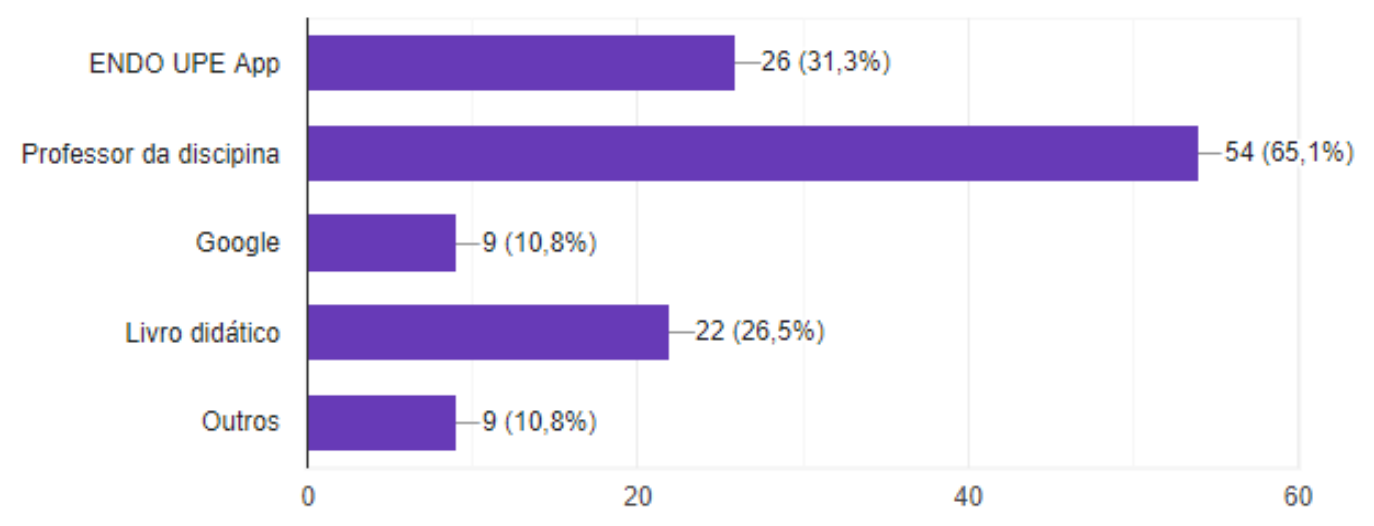

Fonte: Autores. 
A figura em questão foi utilizada para explicitar que 65,1\% dos alunos, ou seja, a maioria, informou que, em relação à temática abertura coronária, consultou primordialmente o professor da disciplina. Em segundo lugar, com 31,3\%, foi o aplicativo ENDO UPE. Logo, demonstrou a boa aceitação dos alunos em relação ao aplicativo.

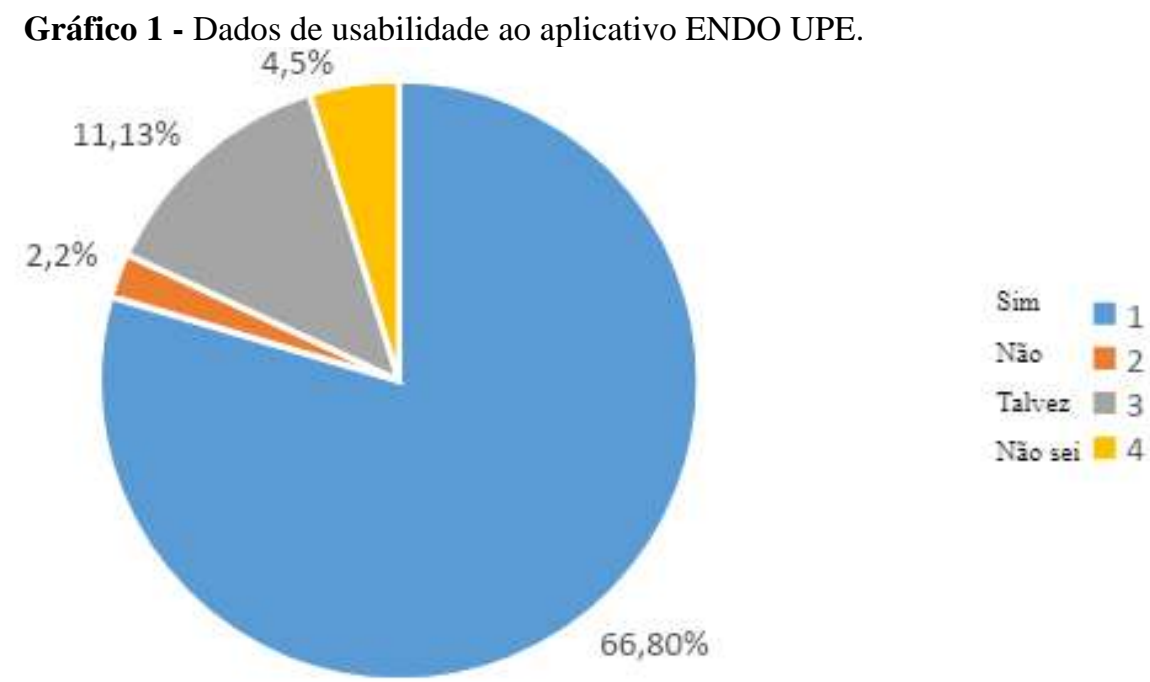

Fonte: Fonte: Autores.

No Gráfico 1, os alunos responderam se utilizariam o aplicativo se tivesse outros assuntos de endodontia: 66 sim; 2 não; 11 talvez e 4 não sei. A partir dessas respostas, entendeu-se que a ferramenta teve uma resposta positiva do corpo discente também para os demais conteúdos da grade curricular.

Gráfico 2 - Possível utilização da aplicação para a vida profissional do entrevistado.

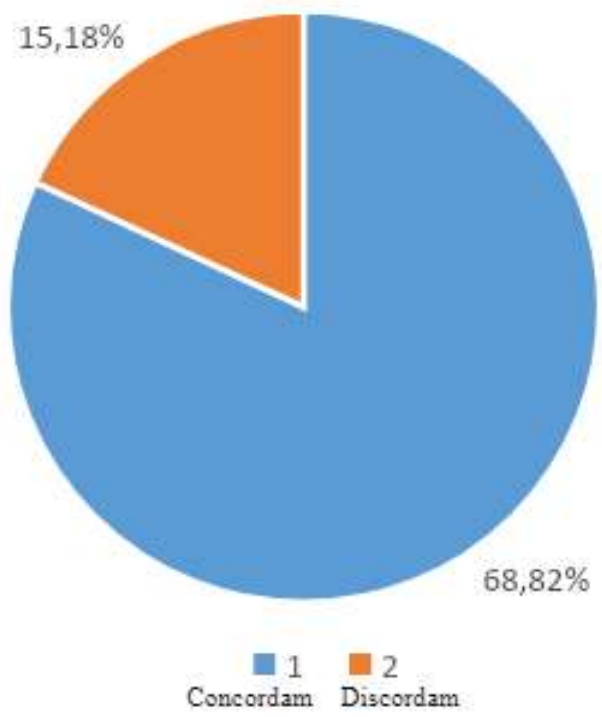

Fonte: Autores.

No segundo gráfico, apresenta a possibilidade dos alunos entrevistados utilizarem o aplicativo na vida profissional: 68 concordaram com a utilização posterior, enquanto 15 discordaram. Sendo assim, a maioria dos entrevistados apontou que a ferramenta poderia ser utilizada também para a prática profissional da Odontologia. 
Tabela 2 - Utilização do aplicativo ENDO UPE pelos estudantes.

\begin{tabular}{|c|c|c|c|}
\hline \multicolumn{4}{|c|}{ Utiliza o aplicativo ENDO UPE } \\
\hline \multirow[t]{2}{*}{ Variáveis } & Sim & Não & p-valor \\
\hline & n $(\%)$ & n $(\%)$ & \\
\hline \multicolumn{4}{|c|}{ Quantidade de aplicativos baixados } \\
\hline 1-4 aplicativos & $2(33,3)$ & $4(66,7)$ & $0,032 *$ \\
\hline 5-7 aplicativos & $10(90,9)$ & $1(9,1)$ & \\
\hline Mais de 7 & $51(77,3)$ & $15(22,7)$ & \\
\hline \multicolumn{4}{|c|}{ Utilização de aplicativos na área de saúde } \\
\hline $\operatorname{Sim}$ & $55(82,1)$ & $12(17,9)$ & 0,019 * \\
\hline Não & $8(50,0)$ & $8(50,0)$ & \\
\hline \multicolumn{4}{|c|}{ Utilização do aplicativo para outros conteúdos de Endodontia } \\
\hline Sim & $51(77,3)$ & $15(22,7)$ & 0,004 * \\
\hline Não & $2(100,0)$ & $0(0,0)$ & \\
\hline Talvez & $10(90,9)$ & $1(9,1)$ & \\
\hline Não sei & $0(0,0)$ & $4(100,0)$ & \\
\hline \multicolumn{4}{|c|}{ O aplicativo será útil na vida profissional } \\
\hline Concorda & $55(80,9)$ & $13(19,1)$ & 0,041 * \\
\hline Discorda & $8(53,3)$ & $7(46,7)$ & \\
\hline
\end{tabular}

Em relação à Tabela 2, foi possível apresentado os dados com associação estatisticamente significativa (p-Valor $\leq$ 0,05) na relação dos estudantes que utilizam o aplicativo ENDO UPE com as variáveis "quantidade de aplicativos baixados", “utilização de aplicativos na área de saúde", "utilização do aplicativo para outros conteúdos de endodontia” e "’o aplicativo será útil na vida profissional".

\section{Discussão}

Neste estudo, observou-se que a maioria dos estudantes $(63,75,4 \%)$, do $3^{\circ}$ ao $5^{\circ}$ ano da Faculdade de Odontologia do Recife, utilizavam o aplicativo ENDO UPE para fins acadêmicos, tendo aprovado seu emprego para o estudo dos procedimentos de abertura coronária, etapa essencial do tratamento endodôntico. Ainda, a maioria disse que utilizaria a ferramenta para a vida profissional (após a graduação).

Diante desta realidade, os aplicativos são recursos que devem ser aproveitados para a inclusão digital educacional (Galvão \& Puschel, 2012). Esta prática acompanha o comportamento cultural da sociedade contemporânea, em permanecer a maior parte do seu tempo conectada à internet, com acesso a informações em tempo real, interagindo não somente em casa, mas em qualquer ambiente e a qualquer hora. (Costa et al., 2015, Costa et al., 2015)

Assim, levantou-se a importância da modificação de metodologias acadêmicas tanto para professores como para estudantes insatisfeitos com os recursos educacionais tradicionais. (Reinaldo et al., 2016)

Independente da marca comercial, todos os estudantes da amostra possuíam smartphones que permitiam $a$ instalação do aplicativo investigado, e durante a pesquisa não foi percebida a necessidade de instrução para seu acesso e gerenciamento. Nesse sentido, 79,5\% já utilizavam mais de sete aplicativos disponíveis em lojas virtuais, em sua maioria operados pelos sistemas Android e IOS, e quase todos (96,8\%) informaram ser uma ferramenta de acesso fácil, simples e rápido. A maioria 
dos usuários de smartphones não precisam de treinamento especializado para seu uso, por acessarem inúmeros aplicativos em diferentes áreas do conhecimento, além de apresentarem a vantagem de serem em sua maioria, gratuitos e extremamente intuitivos. (Reinaldo et al., 2016)

Ou seja, é notável que as TICs proporcionam agilidade e melhora na dinâmica de aprendizado, contudo, alguns professores ainda não sabem usar essas novas tecnologias a seu favor, interrompendo o sistema de modernização do ensino (Souza, 2013). Além disso, pode-se tomar como desafio na inclusão das tecnologias no âmbito educacional a pobre formação acadêmica de alguns docentes quanto à adoção das mídias digitais, a insuficiência da estrutura educacional e o pouco incentivo das políticas públicas em relação ao assunto. (Ferreira et al., 2018)

Como este tipo de mídia se tornou ainda mais atrativa, por permitir o acesso à informação e ao conhecimento sem restrição de tempo e espaço, a elevada utilização do aplicativo ENDO UPE pelos participantes da pesquisa já era esperada, e se confirmou nos resultados desta pesquisa $(75,9 \%)$.

No dia a dia, os estudantes utilizam em especial os aplicativos móveis para compartilhar informações e, principalmente, navegar nas redes sociais, jogos/entretenimento, edição de fotos, ouvir músicas, realizar transações bancárias, acessar mapas, transportes e gerenciar serviços. Porém, também já acessam livros eletrônicos, documentos científicos, blogs, sites e assistem a vídeos educacionais. Resultados de uma revisão da literatura, em 2018, apontaram que as tecnologias estruturadas pelas bases da internet são utilizadas majoritariamente nos cursos de graduação, em pesquisas na literatura científica e para delineamentos epidemiológicos, como também na prática clínica, no acesso a aplicativos para consulta de diagnósticos e terapias. Entre os profissionais, estão sendo muito utilizados para estratégias de administração e marketing e em redes sociais de compartilhamento, como Instagram, Facebook e WhatsApp (Silva et al., 2018).

Neste estudo, observou-se associação estatisticamente significativa entre o uso do aplicativo ENDO UPE e as seguintes respostas: ter de 5 a 7 aplicativos já instalados em seus dispositivos e utilizar aplicativos na área da saúde e educacionais. Já foi documentada na literatura a existência de 306 Apps de conteúdo na área odontológica, sendo 73,9\% para o sistema operacional Android, 23,9\% para o iOS e 2,3\% para ambos os sistemas. A maioria dos Apps são gratuitos (92,5\%) e predominantemente desenvolvidos em língua inglesa (68,3\%). A maior parte são destinados para crianças (46,4\%) e adultos $(51,0 \%)$, sendo poucos para os adolescentes (2,6\%) (Oliveira et al., 2017). No entanto, a produção de aplicativos educacionais não está acompanhando esta tendência, por isso é premente a necessidade de engajamento da comunidade pedagógica para a incorporação desta tecnologia para fins educacionais, pois, embora a popularização dos smartphones entre os professores ainda se encontra em um estágio experimental, para os estudantes continua em franca ascensão (Tibes et al., 2014).

Logo, compreende-se que a incorporação dessas ferramentas pode oferecer ao estudante o controle da sua própria aprendizagem, colocando em prática os argumentos construtivistas das metodologias ativas, nas quais o indivíduo é agente diligente do seu próprio conhecimento e interage de maneira cognitiva e comportamental, com o artefato de mediação que é a tecnologia, estabelecendo a parceria do estudo presencial e online, ou seja, blended learning.

O tema abordado no aplicativo ENDO UPE é importante para a atuação prática do estudante durante o curso de Odontologia, porque contém informações necessárias à prestação de cuidados aos pacientes portadores de patologias pulpares e/ou periapicais que necessitam da intervenção endodôntica de acesso coronário e representa um reforço contextual complementar para o esclarecimento de qualquer dúvida. Este aplicativo foi desenvolvido por docentes da disciplina de endodontia na Faculdade de Odontologia de Pernambuco no ano de 2018 para disponibilizar o conteúdo teórico deste tema por meio de textos, imagens e vídeos, para o estudo prévio pelos estudantes da disciplina de Endodontia da Universidade de Pernambuco, como parte da estratégia de ensino-aprendizagem de sala de aula invertida (flipped classroom).

$\mathrm{Na}$ seleção e elaboração do conteúdo, houve preocupação em oferecer informações suficientes, com clareza nos conceitos e coerência em relação ao tema. O vocabulário foi simples, por meio de textos curtos de fácil leitura, que 
rapidamente promovessem os resultados esperados de aprendizagem, associados às imagens e vídeos, que ilustram o procedimento que será executado pelos estudantes, gerando motivação e melhor compreensão de conceitos. $\mathrm{O}$ aplicativo apresenta uma diversidade de recursos de livre escolha para exploração do estudante, gerando aprendizado de modo significativo, inclusive o resgate de informações relevantes no ato de recordar.

Este estudo revelou que se faz necessária a análise de um paradoxo, pois é senso comum que os estudantes passam muito tempo acessando os dispositivos móveis (smartphones e tablets), todavia com aparente resistência ao uso para fins acadêmicos, pois parte dos entrevistados $(24,1 \%)$ declaram que não fazem uso do aplicativo, $58,6 \%$ dos estudantes relataram que utilizavam o aplicativo ENDO UPE, porém "raramente", e apenas 1,6\% concordaram que usavam "sempre".

Possivelmente essa frequência seria maior caso o conteúdo do dispositivo não tratasse apenas de uma etapa do procedimento endodôntico, pois com exceção do período em que estão cursando a disciplina de Endodontia, a motivação do acesso para estudo ocorre por demanda pessoal e de forma espontânea, quando necessário. Há uma tendência também de haver o decréscimo do uso à medida que o os estudantes dominam o assunto e avançam no curso. De qualquer modo, 98,6\% dos participantes da pesquisa julgaram que o aplicativo é de fácil compreensão, manuseio e navegabilidade, provavelmente por ter sido projetado com cores e fundos simples, e por ícones, como nos demais aplicativos de telefonia móvel. Contudo, iniciativas como esta, devem ser valorizadas, e são estimuladas pelas Diretrizes Curriculares Nacionais para os cursos de Odontologia, aproveitando as tecnologias digitais que já são consideradas instrumentos culturais dessa época, por apresentarem um caráter ubíquo na sociedade contemporânea, proporcionando planejamentos didáticos-pedagógicos que contribuem para a constituição de um sujeito que interage com o individual e o social. (Oliveira, et al., 2017)

Os estudantes também relataram que o aplicativo era de fácil acesso. Esta opinião pode ter sido favorecida por estar disponível em múltiplas plataformas na internet e não apenas para computadores ou mesmo notebooks, de portabilidade mais difícil. Esta abrangência do uso da comunicação móvel celular firma-se como o fenômeno tecnológico recente que melhor atingiu a população, de forma rápida e horizontal, nos últimos tempos.

O laboratório de simulações clínicas foi o local que os estudantes mais acessaram o aplicativo, o que se justifica por ser o ambiente de aulas práticas, onde ocorre o primeiro momento de ensino deste conteúdo, havendo maior interesse para aprendizagem por parte dos estudantes. O segundo local mais utilizado foi o ambulatório de atividades clínicas, onde o estudante aplica o conteúdo aprendido anteriormente. Uma parcela considerável apontou que acessaram em ambos os locais, e em sala de aula $(31,7 \%)$.

Foi constatado, com resultados estatisticamente significativos, que os usuários do aplicativo gostariam que outros conteúdos da endodontia fossem adicionados e que pretendem continuar a acessá-lo mesmo durante a vida profissional. Com base nesta resposta, observou-se que o acesso ao conteúdo fornecido alcançou a satisfação da maioria dos estudantes, de forma se tornou uma ferramenta válida para auxiliar nas demandas acadêmicas dentro e fora do ambiente da universidade, com utilidade durante a vida profissional, pois a realização da abertura coronária está presente corriqueiramente no dia a dia do consultório odontológico.

$\mathrm{Na}$ literatura científica encontram-se outras pesquisas que apresentaram resultados semelhantes em relação à aprovação dos usuários quanto ao uso de aplicativos educacionais em Odontologia, como a experiência desenvolvida para o manejo de pacientes especiais e da ferramenta de apoio ao estudo da anatomia humana (Costa, 2015).

Mesmo diante de experiências exitosas com o uso de aplicativos educacionais, quando os estudantes foram questionados sobre a principal fonte de consulta para o aprendizado do conteúdo abertura coronária, o aplicativo foi apontado em segundo lugar (31,3\%), perdendo colocação para a consulta aos professores da disciplina $(65,1 \%)$. Todos os participantes desta pesquisa concordaram que a presença do professor em sala de aula é essencial. Entretanto, o docente deve se apresentar como facilitador, ajudando na compreensão do assunto e para tirar dúvidas. Embora o uso de novas tecnologias seja, muitas 
vezes, simples e prático, pode ser ineficaz se não houver o domínio da técnica pelo instrutor e o entendimento do processo pelo estudante. (Valente, 2014)

Este contexto apresenta o desafio do professor contemporâneo que deve saber aliar o grande potencial pedagógico destes instrumentos ao papel de mediador do processo de ensino-aprendizagem. Diante deste momento de transição, deve adaptar a utilização de métodos tradicionais e inovadores, e ser o grande responsável pelo desenvolvimento e a condução de estratégias adequadas para melhor abordagem de cada conteúdo especificamente, agregando os benefícios destes e de outros recursos, em qualquer área do conhecimento, tanto para uso em sala de aula, como em cenários de práticas.

A apresentação destes resultados visa contribuir com a educação mediada pelo uso de aplicativos, enfatizando a importância do desenvolvimento racional de ferramentas que busquem uma transformação significativa na educação, apresentando mais um meio para atingir os objetivos educacionais, que acompanhe as evoluções tecnológicas, melhorando a qualidade do ensino com métodos modernos e diversificados.

\section{Conclusão}

Com o trabalho desenvolvido, é possível concluir que as tecnologias precisam estar mais presentes no âmbito educacional, em todas as áreas, visto que as inovações com a incorporação de meios tecnológicos podem trazer vantagens de aprendizado. Para tanto, de início, é preciso quebrar o paradigma de que somente na sala de aula presencial o professor pode construir um conhecimento de qualidade. Os dados colhidos em campo demonstram que os estudantes do curso de Odontologia da Faculdade de Odontologia do Recife (FOR) utilizam de forma ampla o aplicativo ENDO UPE, o reconhecendo como ferramenta educacional do conteúdo de abertura coronária da Endodontia.

Compreendeu-se que há espaço para a implementação dessas ferramentas, de modo que é esperado, em alguns anos, um crescimento ainda maior desse tipo de aplicativo. A aceitação do aplicativo estudado, assim como o reconhecimento dos alunos de que poderia, inclusive, ser ampliado na universidade, mostrou que novas ferramentas podem e devem ser elaboradas em prol do desenvolvimento acadêmico.

\section{Referências}

Barra, D. C. C., Paim, S. M. S., Sasso, G. T. M. D., \& Colla, G. W. (2018). Métodos para desenvolvimento de aplicativos móveis em saúde: revisão integrativa da literatura. Texto \& Contexto-Enfermagem, 26.

Barros, W. C. T. D. S. (2015). Aplicativo móvel para aprendizagem da avaliação do nível de consciência em adultos (OMAC).

Campos, L. F. X. A. (2017). Aplicativo de Saúde Bucal para Educação Infantil.

Costa, B. M. B., Ribeiro Filho, J. M. A., Melo Júnior, P. M. R. de ., Maia, S. M. A. S., Silva, L. B., Oliveira, N. G. de ., Espíndola-Castro, L. F., Isaltino, M. da C., Álvares, P. R., Sousa, J. S. S. S., Diniz, L. L. de A., Melo Filho, D. C. de ., Silva, I. C. da ., Cavalcanti, P. P. A. S., \& Braga, M. L. . (2021). Percepções sobre a plataforma Google Classroom como ferramenta de apoio ao ensino-aprendizagem durante a pandemia da COVID-19. Research, Society and Development, 10(15), e464101521945.

Costa, S. R. S., Duqueviz, B. C., \& Pedroza, R. L. S. (2015). Tecnologias Digitais como instrumentos mediadores da aprendizagem dos nativos digitais. Psicologia Escolar e Educacional, 19, 603-610.

da Costa, R. D. A., de Almeida, C. M. M., de Melo Nascimento, J. M., \& Lopes, P. T. C. (2015). Percepções de acadêmicos sobre o desenvolvimento de um aplicativo para dispositivos móveis como ferramenta de apoio ao ensino e a aprendizagem em anatomia humana. Redin-Revista Educacional Interdisciplinar, 4(1).

Júnior, A. M. L. F., Miranda, N. R., Assunção, R., da Silva, S. A., de Oliveira, F. A. M., \& de Oliveira, R. G. (2016). Percepção de estudantes de Odontologia sobre metodologias ativas no processo de ensino-aprendizagem. Revista da ABENO, 16(3), 66-77.

Galvão, E. C. F., \& Püschel, V. A. A. (2012). Aplicativo multimídia em plataforma móvel para o ensino da mensuração da pressão venosa central. Revista da Escola de Enfermagem da USP, 46, 107-115.

Genari, B., Bemfica, J. R. D., Flach, N., Haddad, D. C., Samuel, S. M. W., \& Abegg, C. (2015). Tecnologias de informação e comunicação na educação da Odontologia: estudo transversal de uma população do sul do Brasil. Revista da ABENO, 15(2), 56-64. 
Research, Society and Development, v. 10, n. 17, e138101723821, 2021

(CC BY 4.0) | ISSN 2525-3409 | DOI: http://dx.doi.org/10.33448/rsd-v10i17.23821

de Melo Júnior, P. M. R., Maia, S. M. A. S., da Cunha, R. S. C., Júnior, E. E., \& Bollela, V. R. (2018). Sala de aula invertida para o ensino do conteúdo abertura coronária em Endodontia. Revista da ABENO, 18(2), 182-191.

LoBiondo-Wood, G., \& Haber, J. (2001). Confiabilidade e validade. Pesquisa em enfermagem: métodos, avaliação crítica e utilização, 4, 186-189.

Lobo, L. C. (2015). Educação médica nos tempos modernos. Revista Brasileira de Educação Médica, 39, 328-332.

Lopes, R. T., Pereira, A. C., \& Silva, M. A. D. D. (2016). Análise comparativa da familiaridade e uso das TIC por alunos de Odontologia. Revista Brasileira de Educação Médica, 40, 254-260.

de Oliveira, A. R. F., \& de Menezes Alencar, M. S. (2017). O uso de aplicativos de saúde para dispositivos móveis como fontes de informação e educação em saúde. RDBCI: Revista Digital de Biblioteconomia e Ciência da Informação, 15(1), 234-245.

Pereira, F. G. F., Frota, N. M., Silva, D. V. D., Sousa, L. M. O. D., \& Almeida, J. C. D. (2017). Avaliação de aplicativo digital para o ensino de sinais vitais. Revista Mineira de Enfermagem, 21.

Reinaldo, F., Magalhães, D., Reis, L., Gaffuri, S., Freddo, A., \& Hallal, R. (2016). Uso de smartphones na educação: Avaliação por grupos focais. CIAIQ2016, 1 .

Ferreira, A., Pauferro, B. C. S., da Cruz, G. M., Trezena, S., \& Batista, R. W. C. (2018). O Uso das Tecnologias de Informação e Comunicação no Ensino e em Odontologia. Revista da AcBO-ISSN 2316-7262, 8(1).

Soares, L. N., \& Cesário, P. M. (2019). Educação híbrida na educação superior. EducVale, 1(2), 72-96.

Souza, R. C. D., Alves, L. A. C., Haddad, A. E., Macedo, M. C. S., \& Ciamponi, A. L. (2013). Processo de criação de um aplicativo móvel na área de odontologia para pacientes com necessidades especiais. Revista da ABENO, 13(2), 58-61.

Tibes, C. M. D. S., Dias, J. D., \& Zem-Mascarenhas, S. H. (2014). Aplicativos móveis desenvolvidos para a área da saúde no Brasil: revisão integrativa da literatura. Revista Mineira de Enfermagem, 18(2), 471-486.

Valente, J. A. (2014). Blended learning e as mudanças no ensino superior: a proposta da sala de aula invertida. Educar em revista, 79-97. 